\title{
Dual effect of the SR proteins ASF/SF2, SC35 and 9G8 on HIV-I RNA splicing and virion production
}

\author{
Sandrine Jacquenet ${ }^{1,2}$, Didier Decimo ${ }^{2}$, Delphine Muriaux ${ }^{2}$ and Jean- \\ Luc Darlix*2
}

Address: ${ }^{1}$ Laboratoire de Médecine et Thérapeutique moléculaire, INSERM CIC9501, 15 rue du Bois de la Champelle, 54500 Vandoeuvre-lèsNancy, France and '2LaboRetro, Unité de Virologie Humaine, INSERM \#412, Ecole Normale Supérieure de Lyon, IFR 128, 46 allée d'Italie, 69364 Lyon cedex 07, France

Email: Sandrine Jacquenet - sandrine.jacquenet@mtm.nancy.inserm.fr; Didier Decimo - ddecimo@ens-lyon.fr; Delphine Muriaux - Delphine.Muriaux@ens-lyon.fr; Jean-Luc Darlix* - jldarix@ens-lyon.fr

* Corresponding author

Published: 22 May 2005

Retrovirology 2005, 2:33 doi:10.1/86/1742-4690-2-33

This article is available from: http://www.retrovirology.com/content/2/I/33

(c) 2005 jacquenet et al; licensee BioMed Central Ltd.

This is an Open Access article distributed under the terms of the Creative Commons Attribution License (http://creativecommons.org/licenses/by/2.0), which permits unrestricted use, distribution, and reproduction in any medium, provided the original work is properly cited.

\begin{abstract}
In HIV-I infected cells transcription of the integrated provirus generates the single full length $9 \mathrm{~kb}$ viral RNA, a major fraction of which is spliced to produce the single-spliced $4 \mathrm{~kb}$ RNAs and the multiple-spliced $2 \mathrm{~kb}$ RNAs. These spliced RNAs are the messengers for the Env glycoproteins and the viral regulatory factors. The cellular SR and hnRNP proteins were shown in vitro to control alternative splicing by binding cis-regulatory elements on the viral RNA. To better understand in vivo the role of the SR proteins on HIV-I genomic RNA splicing and virion production, we used a human cell line expressing high levels of complete HIV-I and either one of the ASF/SF2, SC35, and 9G8 SR proteins. Results show that over-expressing SR proteins caused a large reduction of genomic RNA and that each SR protein modified the viral $9 \mathrm{~kb}$ RNA splicing pattern in a specific mode. In fact, ASF/SF2 increased the level of Vpr RNA while SC35 and $9 \mathrm{G} 8$ caused a large increase in Tat RNA. As expected, overexpressing SR proteins caused a strong reduction of total Gag made. However, we observed by immuno-confocal microscopy an accumulation of Gag at the plasma membrane and in intracellular compartments while there is a dramatic reduction of Env protein made in most cells. Due to the negative impact of the SR proteins on the levels of genomic RNA and HIV-I structural proteins much less virions were produced which retained part of their infectivity. In conclusion, SR proteins can down-regulate the late steps of HIV-I replication.
\end{abstract}

\section{Background}

From a genome of only $9000 \mathrm{nt}$ in length, HIV-1 directs the synthesis of 15 proteins essential for its replication and dissemination (for review see ref. [1]). In order to generate mRNAs required for the synthesis of these proteins, HIV-1 uses the cellular splicing machinery. Through alternative splicing of its primary RNA transcript containing 4 donor sites (D1, D2, D3 and D4) and 8 acceptor sites (A1, A2, A3, A4a, A4b, A4c, A5 and A7), more than 30 different $\mathrm{mRNAs}$ are generated and divided into three classes of $2 \mathrm{~kb}, 4 \mathrm{~kb}$ and $9 \mathrm{~kb}$ in length (Figure 1) [2]. The $2 \mathrm{~kb}$ mRNAs are fully spliced and principally encode the regulatory proteins Tat and Rev and accessory proteins Nef and Vpr. The single-spliced 4 kb RNAs are bicistronic and code for the Env glycoproteins and viral factor Vpu, and the unspliced $9 \mathrm{~kb}$ RNA serves both as mRNAs for the 

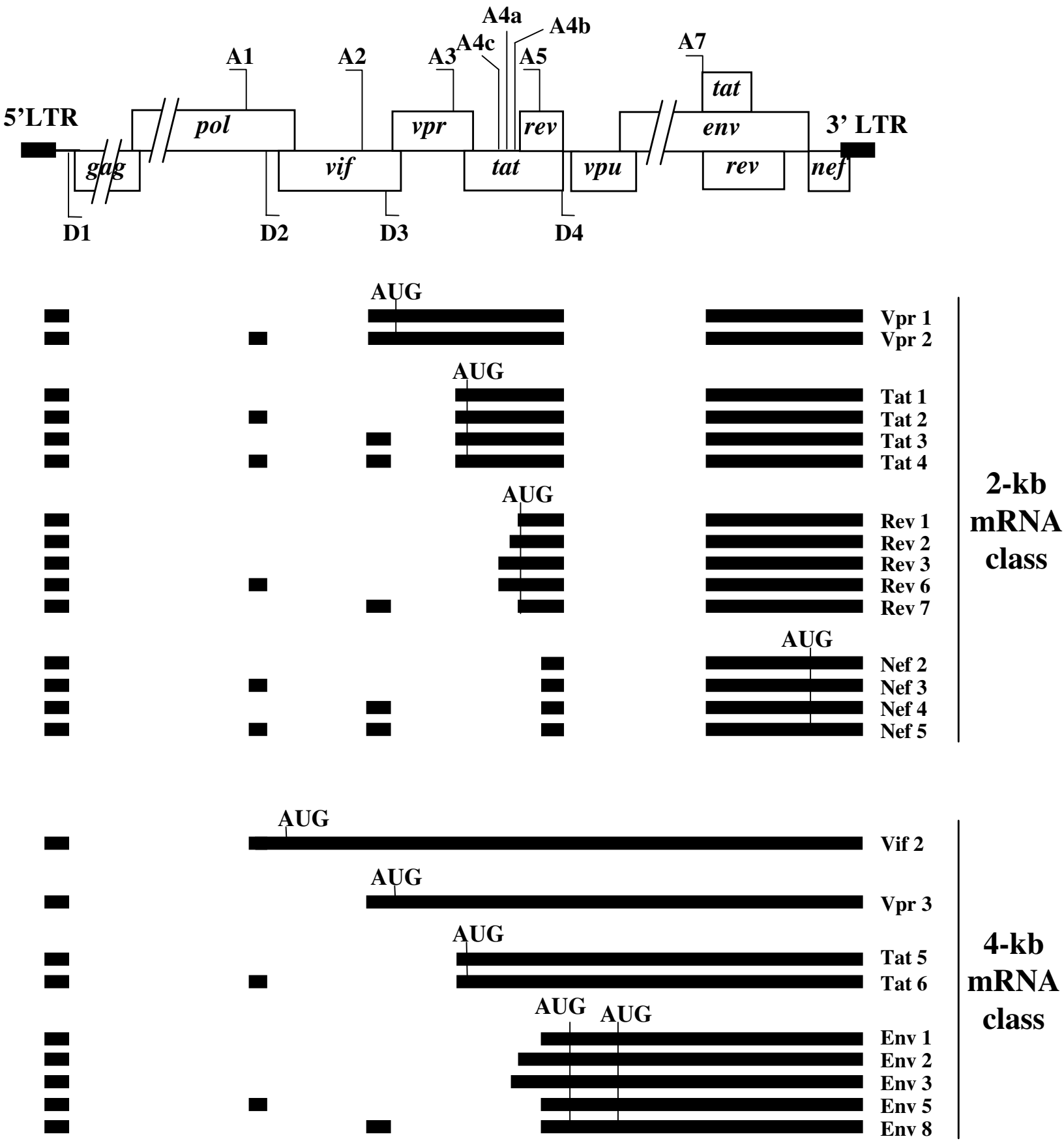

\section{9-kb mRNA}

Figure I

HIV-I splicing pattern. Schematic representation of HIV-I proviral DNA. Open boxes represent the open reading frames encoding the viral proteins. Black boxes represent exons generated by combination of donor sites (DI to D4) and acceptor sites (AI to A7). The viral translation initiator codons are indicated by AUG. 
Gag and Gag-Pol polyproteins as well as pre-genomic RNA for Gag assembly. Rev is crucial because it directs the export of the unspliced and single-spliced mRNAs from the nucleus to the cytoplasm that permits their translation $[3,4]$. A fine tuning of splicing is then critical to ensure the balance between spliced versus unspliced viral RNAs.

HIV-1 splicing regulation relies on the presence of (i) suboptimal splice sites [5,6], (ii) exonic and intronic cis-acting elements [7-15] and (iii) trans-acting factors (generally hnRNPs and SR proteins) that mediate their effects by binding these elements [16-19]. SR proteins belong to a conserved family of structurally and functionally related phosphoproteins (for review, ref. [20]). These proteins participate in constitutive splicing by causing stabilizing interactions with components of the splicing machinery and are able to influence the choice of splicing sites in alternative splicing (for review see ref. [20]). The high level of conservation of the splicing pattern in different HIV expressing cells suggests that splicing regulation is critical for efficient virus replication $[2,21,22]$. Because SR proteins ASF/SF2, SC35, 9G8 and SRp40 have been shown to cause an imbalance in the HIV-1 splicing pattern in vitro and ex vivo [19,23-26], we investigated the impact of SR protein over-expression on virus production and infectivity in a human cell line expressing infectious HIV1 .

In the present study we show that overexpression of one of the three SR proteins ASF/SF2, SC35 and 9G8 together with HIV-1 strongly affected the full length viral RNA splicing pattern, notably resulting in a strong reduction of the genomic RNA and Env mRNA levels. As a consequence, only small amounts of viral particles were produced which, however, retained part of their infectivity.

\section{Results}

\section{SR proteins alter the splicing pattern of HIV-I}

Human cells (293T) were co-transfected by the calcium phosphate precipitation method with $10 \mu \mathrm{g}$ of HIV-1 pNL4-3 [27] and $10 \mu \mathrm{g}$ of irrelevant plasmid pCLacZ (control) or 5-10 $\mu \mathrm{g}$ of one of the SR protein-expression vectors, pXJ41-ASF, pXJ42-PR264 and pXJ42-9G8, encoding respectively ASF/SF2, SC35 and 9G8 proteins [26,28]. Expression of HIV-1 and SR proteins in co-transfected cells was verified by immunoblotting assays (data not shown). We first performed RT-PCR in conditions previously described $[2,29]$ to verify that SR proteins modified HIV-1 splicing pattern as reported elsewhere [26]. Multiple-spliced $2 \mathrm{~kb}$ mRNAs isolated from ASF/SF2 overexpressing cells showed that Vpr1, Tat 2 and Tat 3 were strongly increased as compared with the control (Figures $1,2 \mathrm{~A})$. These observations were confirmed by the analysis of the $4 \mathrm{~kb}$ mRNAs where Tat 6 and Vpr3 mRNAs became the most represented in these conditions probably at the expense of the Env mRNA which proved to accumulate at a low level (Figure 2B). SC35 and 9G8 overexpression led to similar splicing patterns where Tat 1 and Tat 5 mRNAs were the most abundant spliced isoforms (Figures 1, 2). In the case of SC35, splicing was almost completely driven towards Tat 1 production. Because Tat 2 and Tat 6 required splicing at site A1 and Vpr1, Vpr3 and Tat 3 mRNAs at site A2, we concluded that ASF/SF2 participated in a positive regulation of splicing at sites A1 and A2, while SC35 and 9G8 preferentially enhanced splicing at site A3 necessary for Tat mRNA synthesis (Figure 1). These results are in agreement with those obtained in HeLa cells using a truncated non-infectious HIV-1 DNA construct [26] and showed that SR proteins profoundly changed the HIV-1 splicing pattern. However the effects observed in the present experimental conditions were stronger than with the incomplete HIV-1 DNA construct [26].

To further study the SR-mediated commitment of the full length viral RNA to splicing, that is increasing the ratio of viral spliced versus unspliced RNAs, we purified total RNAs from cells expressing HIV-1 and either one of the SR proteins and subjected $10 \mu \mathrm{g}$ total RNA to Northern blot analysis with an HIV-1 env-specific probe. In control HIV1 cells, $8 \%$ of HIV-1 RNA remained unspliced while this amount was lowered to $0.5 \%$ by ASF/SF2 and SC35, and to $1.5 \%$ by $9 \mathrm{G} 8$. This also caused a decrease of total intracellular viral RNAs by two to five fold (Table 1A). We concluded that SR proteins are general activators of HIV-1 splicing, negatively regulating the steady state level of full length viral RNA.

\section{Alterations of HIV-I splicing pattern by SR proteins modify viral protein synthesis}

The profound modifications of the HIV-1 splicing pattern by overexpression of one of the SR proteins were expected to strongly influence viral protein synthesis. Since the unspliced viral RNA serves both as the mRNA for Gag and Gag-Pol synthesis and as the pregenome, we expected the levels of Gag and newly made virions to be strongly reduced by the SR proteins. To this end, levels of intracellular HIV Gag were assessed by CAp24 ELISA on cell lysates $48 \mathrm{~h}$ after DNA transfection (see methods). To measure the levels of virion production, culture supernatants were harvested every day for two days, pooled, clarified by filtration and ultracentrifuged through a $20 \%$ sucrose cushion. Pelleted viral particles were resuspended in TNE buffer (see methods) and virus production was monitored by CAp24 ELISA. Series of measurements indicated that ASF/SF2 and SC35 caused about a 10-12 fold reduction of total Gag synthesized while 9G8 reduced it by roughly 4 fold. These results are in agreement with the relative levels of the unspliced viral RNA in HIV-1 producer cells (Table 1A). 
A: 2-kb mRNA class
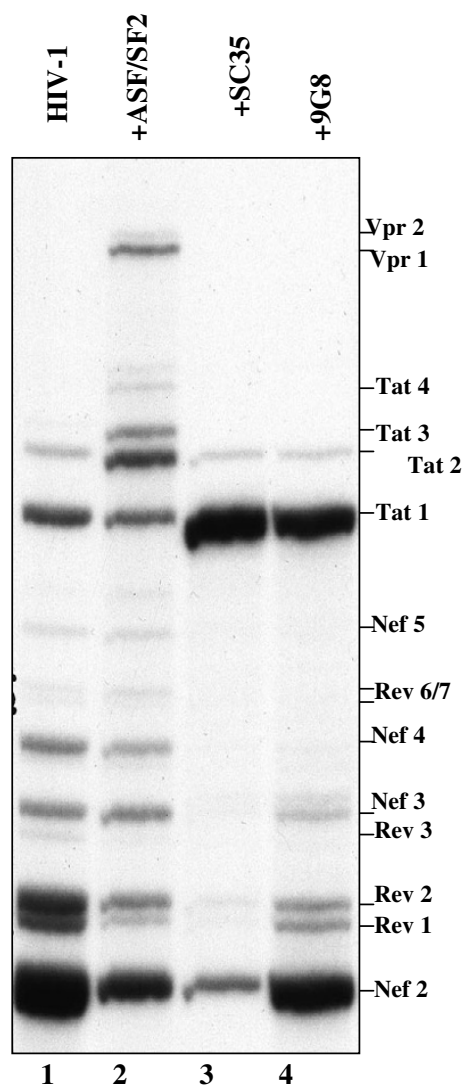

B: 4-kb mRNA class

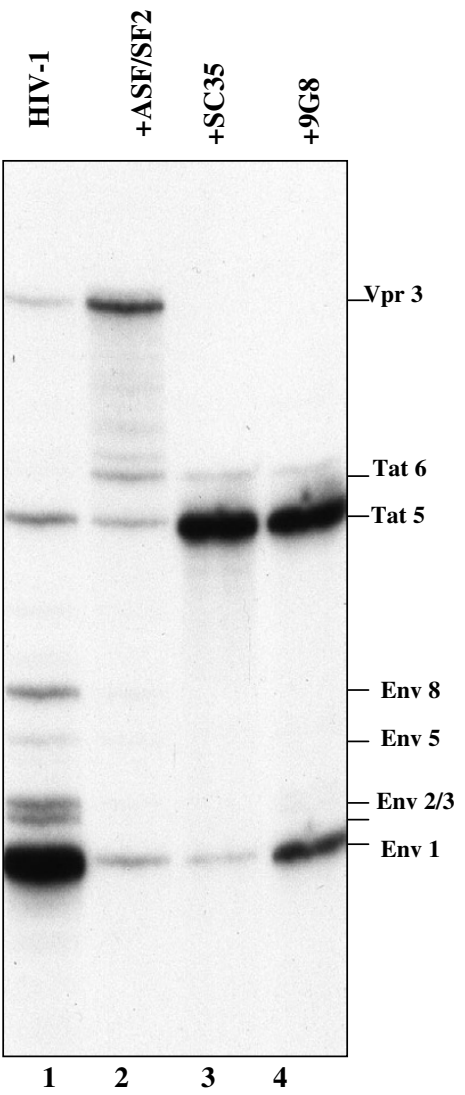

\section{Figure 2}

Regulation of HIV-I alternative splicing by SR proteins. Analysis of $2 \mathrm{~kb}(\mathbf{A})$ and $4 \mathrm{~kb}(\mathrm{~B}) \mathrm{mRNAs}$ was performed by RT-PCR using $10 \mu \mathrm{g}$ of total cellular RNA extracted from 293T cells transfected by HIV-I pNL4.3 only (lane I) or together with one SR plasmid (lanes 2-4). Viral mRNAs were identified according to the nomenclature of Purcell and Martin [2].

Table I: Relative levels of the three viral mRNA classes. The amounts of radioactivy in mRNA signals identified by Northern blotting or by slot blotting experiments (see methods) were measured using a Storm scanner. (A) Relative levels of total intracellular viral RNA were determined as the sum of the radioactivity in the 3 signals corresponding to the 2,4 and 9 kb mRNAs from the same experiments. Levels are expressed as the percentages of total viral RNA in cells transfected with HIV pNL4.3 only used as a reference (100\%) or with HIV-I pNL4.3 and an SR plasmid. For the same degree of DNA transfection, the percentages of the unspliced and spliced mRNAs were calculated relative to the total viral RNA considered as $100 \%$. (B) Values of genomic RNA packaged into a standardized amounts of virions (CAp24 ELISA) are reported relative to the virions produced in the absence of SR protein overexpression (100\%).

\begin{tabular}{lllll}
\hline & \multicolumn{2}{l}{ (A) CELLS } & & (B) VIRIONS \\
\cline { 2 - 5 } & Total & unspliced & spliced & $9 \mathrm{~kb}$ \\
\hline HIV-I (\%) & 100 & 8 & 92 & 100 \\
+ ASF/SF2 (\%) & 25 & 0.5 & 24.5 & 24 \\
+ SC35 (\%) & 20 & 0.5 & 19.5 & 25 \\
+ 9G8 (\%) & 51 & 1.5 & 49.5 & 37 \\
\hline
\end{tabular}


Next we evaluated the relative amounts of cell-associated versus virion-associated Gag. Despite the low levels of total Gag synthesized as measured by CAp24 ELISA, cell-associated Gag was found at unexpected high levels when either one of the SR proteins was overexpressed. Indeed, cellassociated Gag levels were found to be about 40\%, $80 \%$ and even 250\% upon overexpressing ASF, SC35 and 9G8, respectively, as compared with control HIV-1 cells (Figure 3A). Pol was expressed as evidenced by Gag processing and the presence of reverse transcriptase (RT) in the newly formed infectious virions (see below). The pattern of Gag processing by the viral protease was only slightly influenced by overexpressing one of the SR proteins (Figure 3B, compare lanes 2-4 to 1, upper panel).

A large fraction of the $4 \mathrm{~kb}$ mRNAs codes for Env. The very low level of Env glycoproteins present in cells is consistent with the fact that SR proteins strongly reduced the encoding viral mRNA (Figure 2B; Figure 3B bottom panel).

Last we analysed viral protein synthesis directed by the multiple spliced $2 \mathrm{~kb}$ mRNAs, coding for the regulatory proteins Nef and Vpr and the trans-acting factors Tat and Rev. Only the expression of Vpr was found to be markedly enhanced by ASF/SF2 in agreement with the increased level of Vpr mRNAs (Figure 2; Figure 3B, compare lanes 1 and 2; and data not shown).

Thus we can conclude that the SR proteins have a strong indirect impact on viral protein synthesis due to their alterations of the HIV-1 splicing pattern. Only the rather high level of cell-associated Gag appears to contradict this view (see discussion).

\section{Influence of the SR proteins on Gag and Env expression analysed by immuno-confocal microscopy}

To better understand the influence of the SR proteins on Gag and Env synthesis, we examined by immunofluorescence staining and confocal laser microscopy (CLSM), coexpression of the two major viral structural proteins in individual cells. HIV-1 expressing cells were subjected to immuno-staining using anti-MA for Gag (green staining) and anti-gp120 for Env (red staining) antibodies, and all stainings were viewed by confocal microscopy (Figure 4A) (see methods). It is noteworthy that most, if not all, cells co-expressed Gag and Env which accumulated at the plasma membrane and in intracellular vesicles (merge picture in Figure 4A). Co-expression of HIV-1 Gag and Env was confirmed by examining 100 cells where Gag only cells were hardly found, as expected with complete HIV-1 (Figure 5).

Overexpressing ASF/SF2 as evidenced by a blue nuclear staining in most cells (Figure 4B) caused a drastic reduction of Env but only moderately affected Gag (Figure
$4 \mathrm{~B}$, green and red stainings) in agreement with the western blot data (Figure 3B, lane 2). As above, Gag was seen to accumulate in intracellular vesicles and at the plasma membrane while Env was expressed in a heterogeneous manner and mainly located in the cell interior (Figure 4B, HIV Env panel), probably in the Golgi area and in intracellular vesicles (Figure 4B, merge picture). Quantitative values on 100 cells, taking into account that $70-75 \%$ of the cells were positively transfected, showed that coexpression of Gag and ASF was observed in 25\% of the cells while Gag, Env and ASF was seen in only $10 \%$ of the cells. At the same time $65 \%$ of the cells expressed ASF only (Figure 5, bars labelled ASF). These results further showed that the ASF/SF2 SR protein can have a drastic negative impact on HIV-1 since its overexpression caused a nearly complete suppression of Gag and Env expression in a large fraction of the cells (Figures 4B \&5). SC35 (Figure 4C) and 9G8 (not shown) SR proteins had less pronounced effects since a majority of the cells coexpressed Gag and one SR protein (Figure 5; 45 to $55 \%$ see bars labelled gag+SC35 and gag+9G8, respectively) or evenly in the case of Gag, Env and 9G8 (Figure 5; see bar labelled gag+env+9G8). These observations suggest that the SR proteins can have differential effects on HIV-1 structural protein expression.

The influence of the SR proteins on Gag and Env synthesis was further evaluated with respect to virion production and infectivity.

\section{Influence of SR proteins on virion production and infectivity}

This was examined by monitoring the levels of HIV-1 virion production under conditions of increasing expression of the SR proteins. As shown in Figure 6A, SR proteins overexpression induced a dose-dependent inhibition of virion production as compared with control cells co-transfected with HIV-1 pNL4.3 and an irrelevant expression vector. A high dose of SR DNA, notably ASF/SF2, caused a nearly complete inhibition of virion production.

Protein composition of the virions generated by cells overexpressing one of the SR factors, at a HIV-1/SR DNA ratio of 1:0.5, was investigated by western blotting using antibodies against the major core component, CAp24, the RT enzyme, viral factor VPR and the envelope glycoprotein TMgp41. As shown in Figure 6B, CAp24 and RT were found as processed Gag protein and Pol enzyme, respectively, in proportions similar or close to wt HIV-1 particles (see panels labelled $\alpha$ CAp 24 and $\alpha$ RT). On the contrary, VPR was more abundant in virions upon overexpression of ASF/SF2 in agreement with higher levels of the corresponding viral mRNA and protein in cells (Figures 2 and 3B lane 2). With SC35 and 9G8 Vpr was hardly detected in virions in agreement with the very low level of $\mathrm{Vpr}$ 


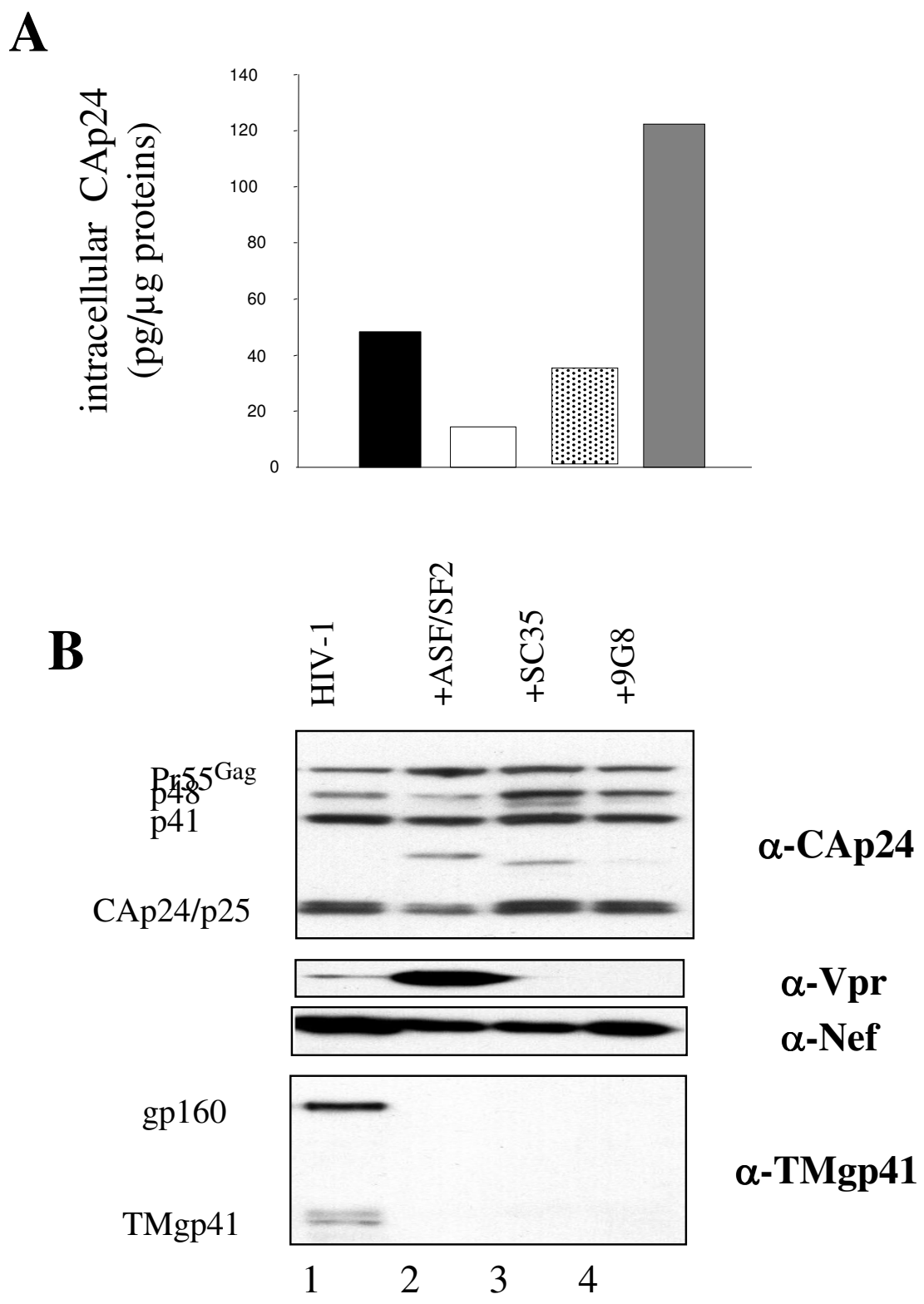

\section{Figure 3}

Influence of SR proteins on HIV-I protein synthesis. 293T cells $\left(2 \times 10^{5}\right.$ per well) were transfected with I $\mu g$ of HIV-I pNL4.3 in the presence of increasing amounts of plasmid encoding either ASF/SF2, SC35 or 9G8. DNA concentrations were maintained constant by supplementation with the $\mathrm{PCLacZ}$ control plasmid which also served to monitor transfection efficiency. Values reported here correspond to assays carried out with a HIV to SR DNA molar ratio of I:05. Cells were recovered two days after DNA transfection. A: Levels of Gag production were assessed by CAp24 antigen ELISA and expressed as pg of CA per $\mu \mathrm{g}$ of total cellular proteins. Note that ASF had a clear negative impact on Gag accumulation in cells whereas $9 \mathrm{G} 8$ had an opposite effect. B: Equivalent amounts of CAp24 antigen as measured by ELISA were subjected to western blotting. The same membrane was alternatively probed with the respective antibodies as indicated on the right: anti-CAp24 for Gag, anti-Vpr for pI5, anti-NEF for p27 and anti-TMgp4I for Env. The viral Gag, Vpr, NEF and Env proteins are indicated according to their molecular weights in kDaltons. Note that SR proteins did not change the Gag processing pattern (compare lanes 2-4 and I). ASF caused an indirect increase of $\mathrm{Vpr}$ cellular accumulation (lane 2) in agreement with its positive effect on $\mathrm{Vpr}$ mRNA level (Figure I). On the other hand SC35 and 9G8 had an opposite effect (lanes 3-4). All Env levels were low (lanes 2-4) except in the control (lane I). 


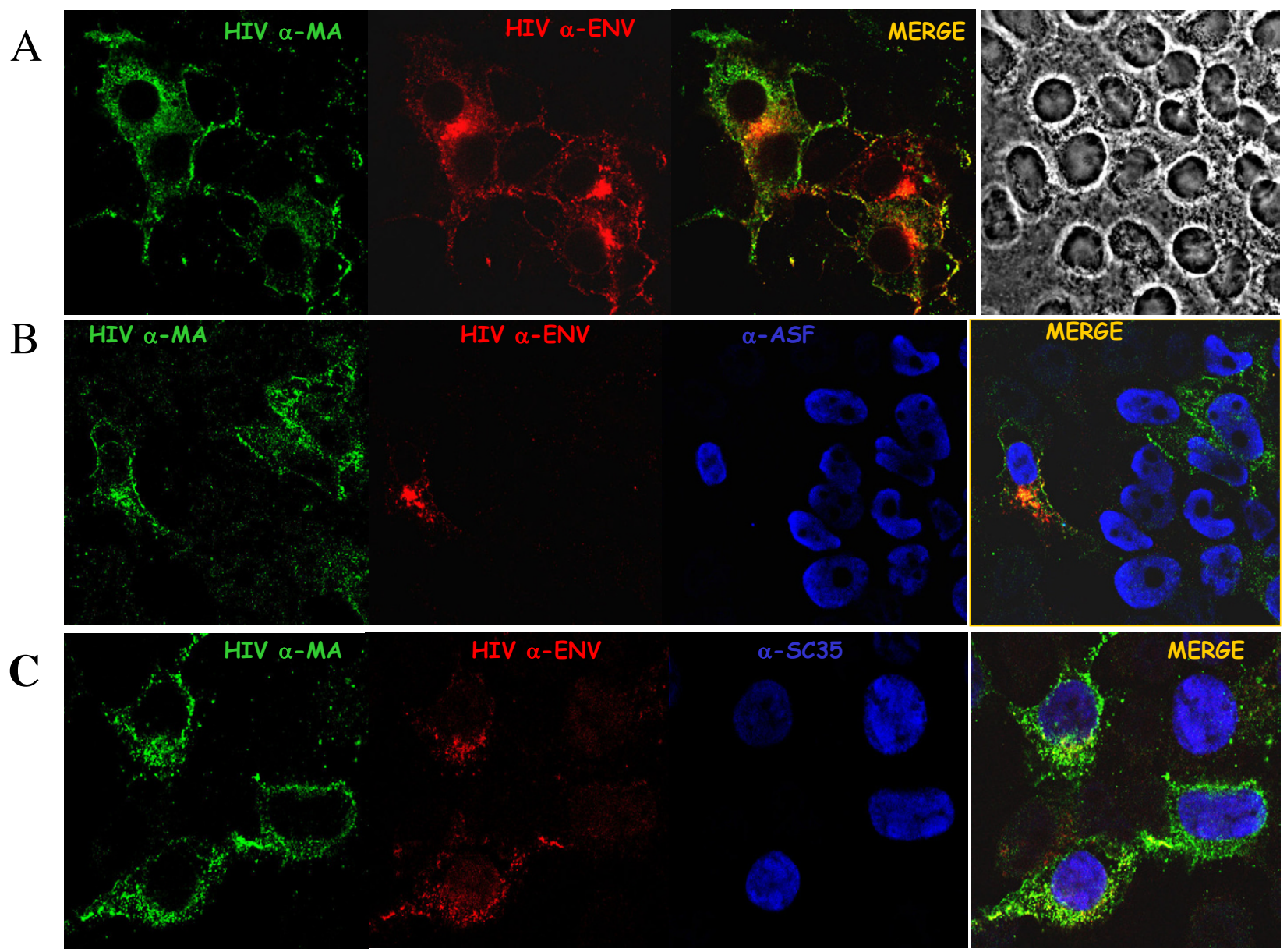

\section{Figure 4}

Confocal microscopy of cells co-expressing HIV-I Gag, Env and SR-protein. Panel A: 293T cells expressing HIV-I pNL4.3 were subjected to immuno-staining using anti-Map 7 (green staining) and anti-Env gp I20 (red staining) antibodies and staining was viewed by confocal microscopy as described in methods. Most if not all cells expressed Gag and Env but only partial colocalization was seen (merge picture). Right panel corresponds to the same cells viewed by phase contrast microscopy. Panel B: same as in A except that His tagged-ASF/SF2 SR protein was overexpressed by DNA transfection with about $75 \%$ transfection efficiency (see methods). ASF/SF2 protein is localized in the nucleus (blue staining) and its overexpression caused a drastic reduction of Env level while Gag remained well expressed in agreement with the western blot data (Figure 3) but with an heterogenous pattern (first panel). Panel C: same as in A except that His tagged-SC35 SR protein was overexpressed by DNA transfection with about 75\% transfection efficiency (see methods). SC35 protein (nuclear blue staining) overexpression caused a reduction of Env level while Gag was still highly expressed in agreement with the western blot data (Figure 2). Note that in all cases examined here (anti-Map I7; green staining in panel A to C) Gag was found to accumulate at the plasma membrane and in intracellular compartments corresponding to vesicles [42] (Muriaux et al., unpublished data).

mRNA and protein in cells (Figure 3B lanes 3-4). All SR proteins examined negatively impacted on the incorporation of Env TMgp41 in virions (Figure 6B, lanes 2-4), again in agreement with the fact that Env mRNA and protein levels were drastically reduced in cells (Figures 2 and $3 \mathrm{~B})$.
To test whether the decreased level of cellular unspliced viral RNA also caused an attenuation of genome packaging into newly made virions, viral particles corresponding to the same amounts of CAp24 were used to purify the genomic RNA which was analyzed by slot-blotting using a 


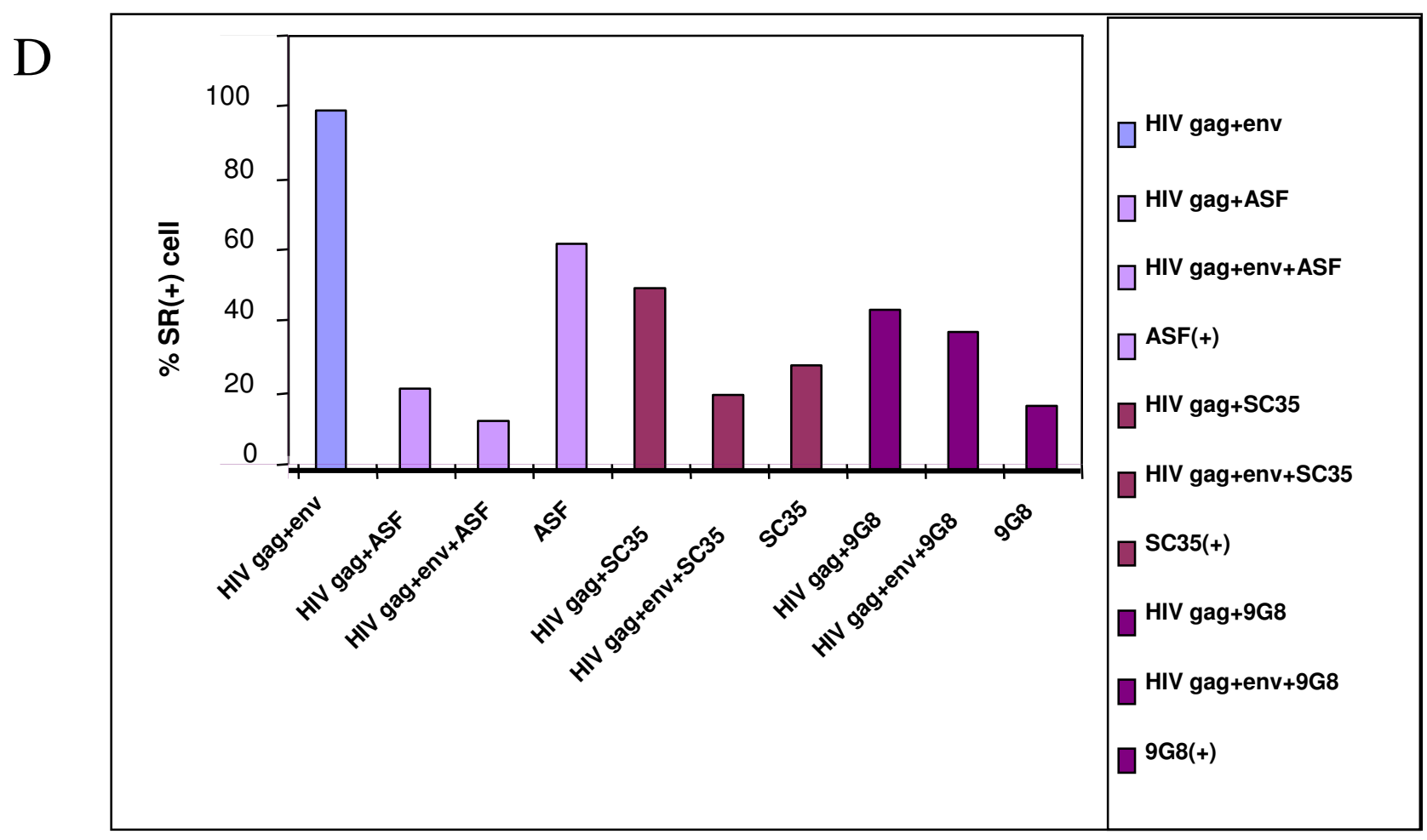

Figure 5

Influence of SR protein on cellular levels of HIV-I Gag and Env. 293T cells expressing HIV-I and one SR protein (either ASF, SC35 or 9G8) were immuno-stained, examined and counted using Confocal Laser Scanning Microscopy (see figure 4). Numbers are representative of more than I00 SR positive cells. For all experiments we evaluated the expression of Gag and Env, and SR protein when applicable. The numbers are expressed as the percentage of all SR positive cells given a DNA transfection efficiency of 70-75\% (not shown). When HIV-I pNL4.3 was transfected alone, $100 \%$ of the cells were found to coexpress Gag and Env (first bar). Upon co-transfection with the ASF coding DNA, a majority of the cells only expressed ASF and about half of them expressed Gag and the SR protein (see ASF bars). Upon co-transfection of pNL4.3 and either the SC35 or 9G8 coding plasmid, a majority of cells expressed Gag and the SR protein (see SC35 and 9 G8 bars).

gag-specific probe. For all overexpressed SR proteins, genomic RNA packaging was reduced from 3 to 4 fold compared with control virions (Table 1B).

To determine the infectivity of virions produced by cells overexpressing one of the SR proteins, the same amount of virus-associated genomic RNA was used to infect Hela P4 cells, a HeLa subtype that constitutively expresses the $\mathrm{CD} 4$ receptor and contains the lac $Z$ gene under the control of the HIV-1 LTR. One day later, blue cells were counted allowing us to assess virus infectivity (see methods). Upon overexpression of each one of the SR proteins, virus infectivity, based on the same amount of genomic RNA, was found to be 30 to $60 \%$ of the control virus, or 6 to 12 fold less based on identical amounts of CAp24-associated particles.

It can be concluded that overexpression of each one of the SR proteins caused a strong reduction of the unspliced viral RNA in cells, and this had a more pronounced effet on virion production than on Gag synthesis (Figures 2, 3, $4,5,6)$. At the same time levels of genomic RNA packaged into progeny virions remained high (Table 1$)$. These findings are in full agreement with the fact that the genomic RNA is considered to be an indispensable partner of Gag in the course of virus assembly. 


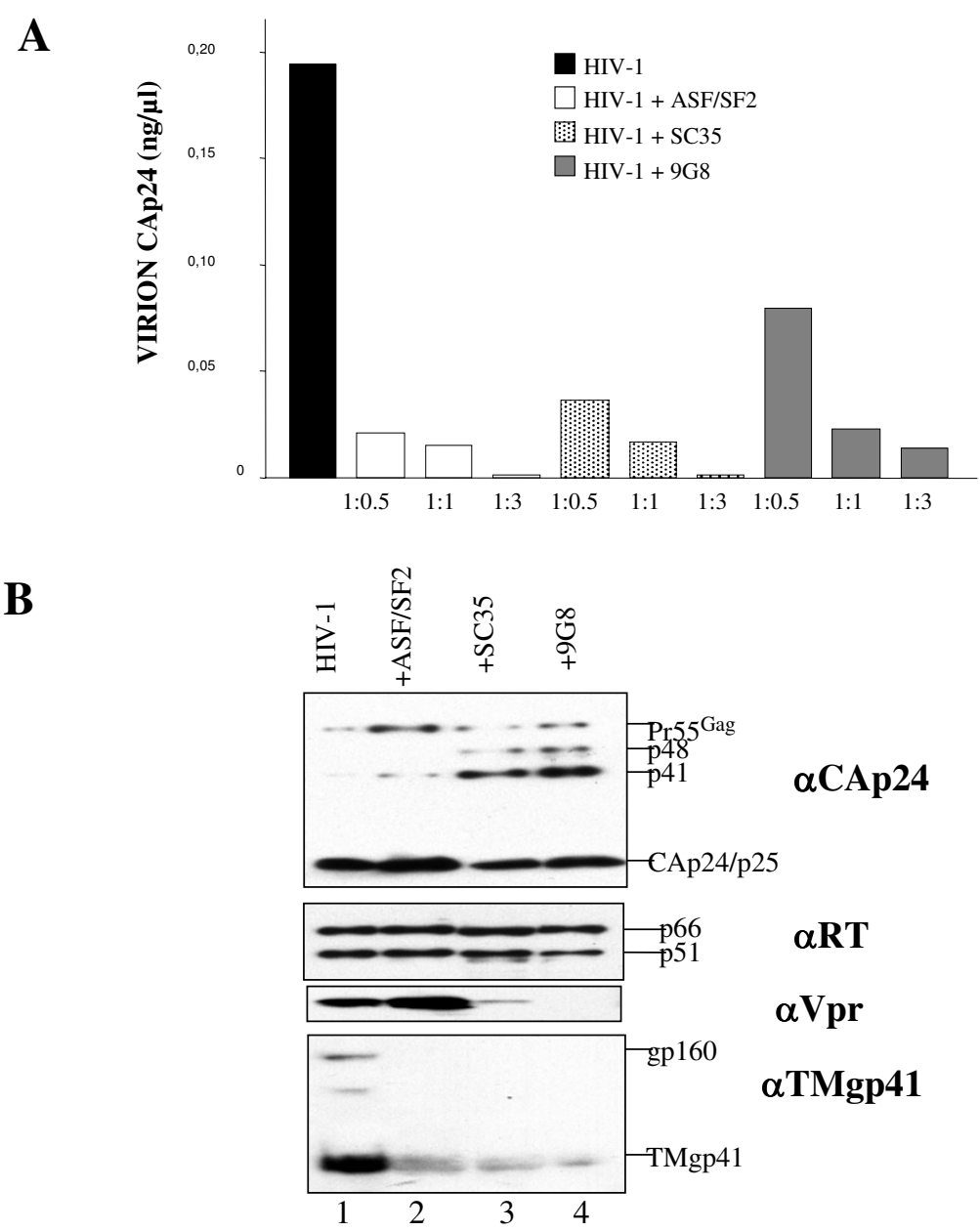

\section{Figure 6}

Expression of viral proteins results from alterations of splicing pattern. 293T cells $\left(2 \times 10^{5}\right.$ per well) were transfected with I $\mu \mathrm{g}$ of HIV-I pNL4.3 in the presence of increasing amounts of plasmid encoding either ASF/SF2, SC35 or 9 G8 (ratios indicate molar amounts of HIV-I DNA vs SR-expressing vector). DNA concentrations were maintained constant by supplementation with the $\mathrm{PCLacZ}$ control plasmid which also served to monitor transfection efficiency. A: Viral production was monitored by CAp24 antigen ELISA and expressed as $\mathrm{ng}$ of p24 per $\mathrm{ml}$ of medium (see methods). Results are representative of 3 independent experiments. Note that the effect of ASF/SF2 on virion production was already drastic at a HIV/SR molar ratio of I:0.5. B: The pelleted viral particles were tested for their content in Gag, Pol, Env and Vpr proteins. Equivalent amounts of CAp24 antigen measured by ELISA were subjected to Western blotting. The same membrane was alternatively probed with the respective antibodies as indicated on the right: anti-CAp24 for Gag, anti-RT for p66 and p5I, anti-Vpr for p 15 and antiTMgp4I for Env. The viral Gag, RT, Vpr and Env proteins are indicated according to their molecular weights in kDaltons. Note that fully mature CAp24 and RTp66/p5 I were abundant in all virion preparations. ASF caused an indirect increase of Vpr incorporation in virions (lane 2) whereas SC35 and 9G8 had an opposite effect (lanes 3-4). All Env levels were low (lanes 2-4) except in the control (lane I).

\section{Discussion}

In the present study, we show that the overexpression of either one of three different SR proteins, namely ASF/SF2, SC35 and 9G8, profoundly affected the HIV-1 splicing pattern (Figure 1) [26], resulting in a drastic decrease of virus production. However, the progeny virions still made retained part of their infectivity. SR protein overexpression caused an oversplicing of the HIV-1 full length transcript and confirm that the targets of activation depend on the SR protein overexpressed. Indeed, ASF/SF2 
stimulates splicing at sites A1 and A2, while SC35 and 9G8 preferentially enhance splicing at site A3 (Figures 1, 2 ). In addition to being general activators of constitutive splicing, results reported here confirm that each one of the three SR proteins exerts specific effects on the alternative splicing of HIV-1 primary RNA transcript (Figure 2) [26].

Little is known about the HIV-1 A1 site. Here, we show that ASF/SF2 participates in the utilization of A1 by a mechanism that requires further investigations. Many elements act in concert to repress splicing at site A2 such as its intrinsic weakness $[5,6]$ and the existence of the hnRNP A/B dependent ESSV located in the noncoding exon flanking sites A2 and D3 [8,9]. Then how does ASF/ SF2 exert this control ? The exon bridging hypothesis proposes that U1 snRNP binding to the downstream donor site acts to increase splicing efficiency at the upstream flanking acceptor site (for review see [20]). Also, SR proteins are known to stabilize U1 snRNP binding on suboptimal donor sites. Consequently, one can imagine that ASF/SF2 reinforces splicing at site A2 by stabilizing spliceosomal interactions at the suboptimal site D3. Accordingly, other SR proteins like SC35 or 9G8 would be expected to have the same effect as ASF/SF2 on site A2 and thus on $\mathrm{Vpr}$ mRNA synthesis. This prediction is inconsistent with our data (Figure 3 ) since overexpression of SC35 and 9G8 did not increase Vpr RNA level. Another possibility is that ASF/SF2 positively regulates splicing at site A2 by counteracting the effect of ESSV. ESSV represses splicing at site A2 by binding cellular hnRNP A/B proteins $[8,9]$. This binding prevents the assembly of U2AF on the polypyrimidine tract (PPT) and subsequently the formation of a functional spliceosome between sites D1 and A2 [9]. SR proteins are thought to also activate weak acceptor sites by facilitating the recruitment of U2AF on the PPT [20]. It is tempting to speculate that the ratio between hnRNP A/B and ASF/SF2 bound close to site A2 modulates the binding of U2AF at this site. This effect of SR proteins is generally mediated by a splicing enhancer, but whether an ASF/SF2-dependent splicing element is required here remains to be determined.

Site A3 is used to generate Tat mRNAs. Like A2, A3 is intrinsikly weak and repressed by hnRNP A/B-dependent ESS2 and hnRNP H-dependent ESS2p $[17,29]$. Our results show that a strong positive control is exerted by SR proteins SC35 and 9G8 at this level. Findings on SC35 are consistent with the recent data of Zahler et al. [19] reporting a novel ESE downstream of A3 that reinforces A3 utilization in the presence of a high level of SC35 in vitro. In addition, hnRNPs act in a trans-dominant manner to counteract that of SC35 in vitro [19]. Taken together these results strongly suggest that changing the hnRNP/SC35 ratio probably leads to activation or repression of splicing at site A3. Little is known on the implications of SR pro- tein 9G8 in HIV-1 splicing. The present data show that 9G8 appears to function in a way similar to SC35 (Figure 2). As for ASF/SF2, the exon bridging hypothesis can be mentioned but 9G8 acts mainly by binding specific cisacting RNA elements [30]. Even if overexpression of SC35 and 9G8 caused a large accumulation of Tat mRNAs, it is likely that these two SR proteins act by distinct mechanisms. Indeed, firstly SELEX experiments showed that 9G8 and SC35 recognize different consensus RNA sequences [30]. Secondly, ESS2 mutations that in vitro strongly reinforce the binding of SC35 on an ESS2-containing transcript have no effect on 9G8 binding to the same substrate $[19,30]$. Sequences important for 9G8 splicing activation remain to be determined.

In conclusion, the exact molecular mechanisms by which high levels of SR proteins cause a strong enhancement of genomic RNA splicing and consequently a severe inhibition of HIV-1 virion production remain to be determined. This is presently under investigation.

As expected, the profound changes of the HIV-1 splicing pattern caused by the overexpression of one of the SR proteins ASF/SF2, SC35 or 9G8 inhibited viral protein synthesis, notably that of the structural proteins Gag and Env (Figures 3, 4, 5) and consequently, virion production (Figure 6A). Still in agreement with such alterations of the HIV-1 splicing pattern (Figures 1, 2), Vpr synthesis was upregulated by ASF whereas SC35 and 9G8 had an opposite effect (Figure 3B). But it was surprising to find a rather high heterogeneous level of cell-associated Gag (Figure 3A). To explain this apparent discrepancy, one should remember that the unspliced viral RNA performs two essential functions, firstly as the mRNA for Gag and GagPol synthesis and secondly as the pregenome for Gag assembly (reviewed in ref. [31]). In fact, the Gag assembly process requires two platforms that are the genomic RNA through specific NC-genomic RNA interactions [31,32] and a cellular membrane in which Gag is anchored via MA-membrane interactions (reviewed in ref. [33]). Membranes are not limiting whereas the full length RNA is probably limiting due to its mobilization by the translating ribosomes (reviewed in ref. [34]). Actually, the fate of the full length viral RNA appears to result from a subtle balance between Gag translation on ribosomes and core assembly governed by Gag-genomic RNA interactions (reviewed in refs [31,35]). In the presence of high amounts of the SR proteins, the unspliced viral RNA is even more limiting and thus probably rarely available for assembly. Hence, the cell-associated Gag corresponds to newly made free Gag molecules as well as Gag in newly assembled core nucleocomplexes which accumulate in the cell (Figure 4) before being released. According to this scheme of virus assembly, the low levels of the pregenomic RNA (Table 1A) and Env (Figure 3B) upon SR over- 
expression may very well explain why Gag assembly and virion release are most probably slowed down (Figure $6 \mathrm{~A})$. In agreement with this interpretation, the level of packaged genomic RNA into newly formed viral particles was decreased by 65 to $75 \%$ (Table 1B). Also in agreement with the above interpretation is the observation that production of high titer lentivectors necessitates expression of the recombinant viral RNA at high levels in vector producing cells [36].

Despite the low level of incorporated Env (Figure 6), virions produced by cells overexpressing one SR protein retained part of their infectivity on Hela P4 cells. This was not unexpected since only a minimal amount of Env appears to be required to drive infectivity in certain model cell systems [37].

\section{Conclusion}

In summary, the data presented here show that elevated concentrations of SR proteins in HIV-1 expressing cells differentially affected viral RNA and protein expression, resulting in a strong decrease of viral progeny made. Thus one can speculate that the coordinated regulation of HIV1 splice site utilization by SR proteins is of critical importance to maintain high levels and balanced ratios of the viral RNAs and hence of the viral proteins made in order to direct optimal virus assembly and production. Thus, HIV-1 probably needs to interact with the splicing machinery. In accordance with this view, SC35 is up-regulated and 9G8 down-regulated in HIV-1 infected cells $[38,39]$. On a more general basis it has been found that SR proteins influence expression and replication of other viruses such as human papilloma virus type 16 [40] and Adenovirus [41].

\section{Materials and methods \\ Plasmids}

Plasmids pXJ41-ASF, pXJ42-PR264 and pXJ42-9G8 [28] that respectively encode ASF/SF2, SC35 and 9G8 in eukaryotic cells were provided by J. Stevenin and R. Gattoni (IGBMC Strasbourg, France). The HIV-1 molecular clone is pNL4.3 (GenBank \#M19921) [27].

\section{Cell cultures, transfections and infections}

HeLa P4 (provided by P. Charneau) and 293T cells (provided by Genethon) were maintained in Dulbecco's modified Eagle's medium supplemented with $10 \%$ fetal calf serum, $2 \mathrm{mM}$ glutamine and antibiotics (penicillin-streptomycin; Invitrogen). One day before transfection, $3 \times 10^{6}$ 293 cells were inoculated in 10-cm Petri dish (except for experiments of Figure 1, see legend). One day later, cells were transfected with $10 \mu \mathrm{g}$ of proviral pNL4.3 and 4-10 $\mu \mathrm{g}$ of SR-expressing vector or of control plasmid pCLacZ by the calcium phosphate precipitation technique according to manufacturer instructions (Gibco). As observed by immuno-staining and confocal microscopy, more than $70 \%$ of the cells were positively transfected. After $12 \mathrm{~h}$, cell culture supernatants were substituted by fresh culture medium. Forty-eight hours after transfection, supernatants were harvested, clarified by filtration through 0.8 $\mu \mathrm{m}$-pore size filters and ultracentrifuged through a $20 \%$ sucrose cushion. Pelleted viruses were resuspended in TNE buffer (25 mM Tris HCl pH 7.5; 150 mM NaCl; $1 \mathrm{mM}$ EDTA). Virus production was monitored in cell culture supernatants and in virus pellets with a CAp24 ELISA capture assay (kindly provided by Valérie Cheynet and Bernard Mandrand, BioMérieux).

Viral titrations were performed by infection of HeLa P4 cells $\left(1.5 \times 10^{5}\right.$ cells per well of a 24 -well plate $)$ with purified viruses containing $1 \mathrm{ng}$ of genomic RNA. After $24 \mathrm{~h}$, cells were fixed and incubated in the presence of $\mathrm{X}$ Gal substrate at $37^{\circ} \mathrm{C}$ until blue color development was complete. Viral titers were determined by counting the number of blue cells in threee different wells.

\section{RNA isolation, Northern blotting and RT-PCR}

Transfected cells were harvested $48 \mathrm{~h}$ after transfection and washed in phosphate-buffered saline (PBS). Twothird of cells were resuspended in PBS and total cellular RNA was extracted with TRIzol reagent as recommended by the manufacturer (Invitrogen). Culture supernatants were treated as indicated above and the level of CAp24 antigen measured by ELISA. Northern blotting of intracellular viral RNAs was performed with $10 \mu \mathrm{g}$ of total RNA and slot-blot of virion-associated genomic RNA was performed with $10 \mathrm{ng}$ of CAp24 antigen. After transfer onto a Hybond-N+ membrane (Amersham Pharmacia Biotech), viral RNAs were probed with radiolabeled fragments from Env for Northern blot and from Gag region for slot-blot. All the mRNAs species were quantified by Storm (Amersham).

The splicing products were analyzed by RT-PCR as previously described [10], except that the forward PCR primer was Odp045 [2]. The PCR products were fractionated on a $6 \%$ acrylamide-7M urea electrophoresis gel and autoradiographed. Individual HIV-1 mRNA species were named according to the nomenclature of Purcell and Martin [2].

\section{Immunoblotting}

One-third of transfected cells were washed in PBS and lysed in PBS containing 0.5 \% Triton. After CAp24 ELISA measurement in cell lysates and in virus pellets, samples were added to $3 \mathrm{X}$ gel loading buffer $(0.5 \mathrm{M}$ Tris- $\mathrm{HCl} \mathrm{pH}$ 6.8; $0.8 \%$ SDS; $40 \%$ glycerol; $5 \% \beta$-mercaptoethanol; $0.03 \%$ bromophenol blue). For immunoblotting, samples containing equal amounts of CAp24 antigen were loaded on a $10 \%$ SDS-PAGE and fractionated proteins were transferred onto a Hybond P membrane (Amersham 
Pharmacia Biotech). Viral proteins were probed with monoclonal anti-CAp24 (BioMérieux), polyclonal antiVpr (\#3951, NIH USA), polyclonal anti-Nef (\#331, NIH USA) or monoclonal anti-TMgp41 (Ab 41A9; Hybridolab, Pasteur) antibodies. The bound antibodies were detected with peroxidase-conjugated anti-mouse IgG antibodies and visualized by the SuperSignal West Pico Chemiluminescent Substrate (Pierce).

\section{Immunofluorescence staining and confocal microscopy imaging}

Transfected 293 T cells were grown on poly-lysine coated coverglass dishes and fixed $24 \mathrm{~h}$ post transfection in $3 \%$ paraformaldehyde (diluted in Phosphate Buffer Saline PBS) for $20 \mathrm{~min}$. The fixative was then removed and the free aldehydes were quenched with $50 \mathrm{mM} \mathrm{NH}_{4} \mathrm{Cl}$. Cells were then permeabilized using $0.2 \%$ Triton X-100 for 5 min and blocked in 1\% BSA. The fixed cells were incubated for one hour at room temperature with primary antibodies: rabbit anti-MAp17 (NIH, USA), human antiHIV-1 gp120 Mab(b12) (NIH, USA), rabbit anti-His for His-tagged 9G8 and ASF proteins, and mouse anti-HA1 for HA-tagged SC35 protein (Sigma). The corresponding fluorescent Alexa ${ }^{\circledast}$ 488, 546 and 633-conjugated secondary antibodies were used at $0.5 \mu \mathrm{g} / \mathrm{ml}$ (Molecular probes). Coverslips were washed three times with PBS and mounted on microscope slides with Mowiol (Sigma). Images were acquired on Axioplan 2 Zeiss CLSM 510 confocal microscope with Argon 488/458, HeNe 543, HeNe 633 lasers and plan apochromat $63 \times 1.4$ oil Ph3 objective, supplied with LSM 5103.4 software.

\section{Abbreviations used}

HIV-1, Human Immunodeficiency Virus type 1.

CAp24, viral capsid protein p24.

MAp17, viral matrix protein p17.

RT, reverse transcriptase.

SR, splicing regulatory proteins.

hnRNP proteins, heterogenous ribonucleoparticle proteins.

Site A, splicing acceptor site. Site D, splicing donor site.

Kb, kilobases. WT, wild type.

PBS, Phosphate Buffer Saline.

BSA, bovine serum albumin.

Mab, monoclonal antibody.

\section{Authors' contributions}

SJ carried out analyses on SR protein-mediated effect on HIV-1 RNA splicing.

DD was in charge of cell culture and transfection assays. DM performed immuno-confocal microscopy experiments and analyses. JLD is the lab head and arranged the manuscript.

\section{Competing interests}

The author(s) declare that they have no competing interests.

\section{Acknowledgements}

We are grateful to J. Stevenin and R. Gattoni for their gifts of anti-ASF/SF2, anti-SC35 and anti-9G8 antibodies and for the SR protein expression plasmids, B. Mandrand (CNRS BioMérieux) for anti-CAp24 ELISA and the NIH (USA) for reagents. Work supported by ANRS, Sidaction and the European TRIoH Consortium. SJ was the recipient of an ANRS fellowship.

\section{References}

I. Frankel AD, Young JA: HIV-I: fifteen proteins and an RNA. Annu Rev Biochem 1998, 67:I-25.

2. Purcell DF, Martin MA: Alternative splicing of human immunodeficiency virus type I mRNA modulates viral protein expression, replication, and infectivity. J Virol 1993, 67(II):6365-6378.

3. Felber BK, Hadzopoulou-Cladaras M, Cladaras C, Copeland T, Pavlakis GN: rev protein of human immunodeficiency virus type $I$ affects the stability and transport of the viral mRNA. Proc Natl Acad Sci U S A 1989, 86(5): I495-1499.

4. Malim MH, Hauber J, Le SY, Maizel JV, Cullen BR: The HIV-I rev trans-activator acts through a structured target sequence to activate nuclear export of unspliced viral mRNA. Nature 1989, 338(62 I 2):254-257.

5. Damier L, Domenjoud L, Branlant C: The DI-A2 and D2-A2 pairs of splice sites from human immunodeficiency virus type I are highly efficient in vitro, in spite of an unusual branch site. Biochem Biophys Res Commun 1997, 237(1):182-187.

6. O'Reilly MM, McNally MT, Beemon KL: Two strong 5' splice sites and competing, suboptimal 3 ' splice sites involved in alternative splicing of human immunodeficiency virus type I RNA. Virology 1995, 21 3(2):373-385.

7. Amendt BA, Si ZH, Stoltzfus CM: Presence of exon splicing silencers within human immunodeficiency virus type I tat exon 2 and tat-rev exon 3: evidence for inhibition mediated by cellular factors. Mol Cell Biol I995, I 5(8):4606-46I5.

8. Bilodeau PS, Domsic JK, Mayeda A, Krainer AR, Stoltzfus CM: RNA splicing at human immunodeficiency virus type I 3 ' splice site $A 2$ is regulated by binding of hnRNP A/B proteins to an exonic splicing silencer element. J Virol 200 I, 75 (18):8487-8497.

9. Domsic JK, Wang Y, Mayeda A, Krainer AR, Stoltzfus CM: Human immunodeficiency virus type I hnRNP A/B-dependent exonic splicing silencer ESSV antagonizes binding of U2AF65 to viral polypyrimidine tracts. Mol Cell Biol 2003, 23(23):8762-8772.

10. Jacquenet S, Ropers D, Bilodeau PS, Damier L, Mougin A, Stoltzfus CM, Branlant C: Conserved stem-loop structures in the HIV-I RNA region containing the A3 3' splice site and its cis-regulatory element: possible involvement in RNA splicing. Nucleic Acids Res 200I, 29(2):464-478.

II. Marchand V, Mereau A, Jacquenet S, Thomas D, Mougin A, Gattoni R, Stevenin J, Branlant $C$ : A Janus splicing regulatory element modulates HIV-I tat and rev mRNA production by coordination of hnRNP Al cooperative binding. J Mol Biol 2002, 323(4):629-652.

12. Si Z, Amendt BA, Stoltzfus CM: Splicing efficiency of human immunodeficiency virus type I tat RNA is determined by both a suboptimal $3^{\prime}$ splice site and a 10 nucleotide exon 
splicing silencer element located within tat exon 2. Nucleic Acids Res 1997, 25(4):86I-867.

13. Staffa A, Cochrane A: Identification of positive and negative splicing regulatory elements within the terminal tat-rev exon of human immunodeficiency virus type I. Mol Cell Biol 1995, I 5(8):4597-4605.

14. Tange TO, Damgaard CK, Guth S, Valcarcel J, Kjems J: The hnRNP Al protein regulates HIV-I tat splicing via a novel intron silencer element. Embo J 200I, 20(20):5748-5758.

15. Tange TO, Kjems J: SF2/ASF binds to a splicing enhancer in the third HIV-I tat exon and stimulates U2AF binding independently of the RS domain. J Mol Biol 200I, 3 I 2(4):649-662.

16. Amendt BA, Hesslein D, Chang LJ, Stoltzfus CM: Presence of negative and positive cis-acting RNA splicing elements within and flanking the first tat coding exon of human immunodeficiency virus type I. Mol Cell Biol 1994, I 4(6):3960-3970.

17. Caputi M, Mayeda A, Krainer AR, Zahler AM: hnRNP A/B proteins are required for inhibition of HIV-I pre-mRNA splicing. Embo J 1999, I 8(14):4060-4067.

18. Caputi M, Zahler AM: SR proteins and hnRNP H regulate the splicing of the HIV-I tev-specific exon 6D. Embo J 2002, 2I(4):845-855.

19. Zahler AM, Damgaard CK, Kjems J, Caputi M: SC35 and heterogeneous nuclear ribonucleoprotein $A / B$ proteins bind to a juxtaposed exonic splicing enhancer/exonic splicing silencer element to regulate HIV-I tat exon 2 splicing. I Biol Chem 2004, 279(II): 10077-10084.

20. Graveley BR: Sorting out the complexity of SR protein functions. Rna 2000, 6(9): || $197-121 \mid$.

21. Gorry PR, Howard JL, Churchill MJ, Anderson JL, Cunningham A, Adrian D, McPhee DA, Purcell DF: Diminished production of human immunodeficiency virus type $I$ in astrocytes results from inefficient translation of gag, env, and nef mRNAs despite efficient expression of Tat and Rev. I Virol 1999, 73(I):352-36I.

22. Sonza S, Mutimer HP, O'Brien K, Ellery P, Howard JL, Axelrod JH, Deacon NJ, Crowe SM, Purcell DF: Selectively reduced tat mRNA heralds the decline in productive human immunodeficiency virus type $I$ infection in monocyte-derived macrophages. J Virol 2002, 76(24):|26||-|262|.

23. Fu XD: Specific commitment of different pre-mRNAs to splicing by single SR proteins. Nature 1993, 365(644I):82-85.

24. Krainer AR, Conway GC, Kozak D: The essential pre-mRNA splicing factor SF2 influences 5 ' splice site selection by activating proximal sites. Cell 1990, 62(I):35-42.

25. Mayeda A, Screaton GR, Chandler SD, Fu XD, Krainer AR: Substrate specificities of SR proteins in constitutive splicing are determined by their RNA recognition motifs and composite pre-mRNA exonic elements. Mol Cell Biol 1999, 19(3): 1853-1863.

26. Ropers D, Ayadi L, Gattoni R, Jacquenet S, Damier L, Branlant C, Stevenin J: Differential effects of the SR proteins 9 G8, SC35, ASF/ SF2 and SRp40 on the utilization of the AI to A5 splicing sites of HIV-I RNA. J Biol Chem 2004, 279:29963-29973.

27. Adachi A, Gendelman HE, Koenig S, Folks T, Willey R, Rabson A, Martin MA: Production of acquired immunodeficiency syndromeassociated retrovirus in human and nonhuman cells transfected with an infectious molecular clone. J Virol 1986, 59(2):284-29l.

28. Bourgeois CF, Popielarz M, Hildwein G, Stevenin J: Identification of a bidirectional splicing enhancer: differential involvement of SR proteins in ' $\mathbf{~}^{\prime}$ or ' $\mathbf{3}^{\prime}$ splice site activation. Mol Cell Biol 1999 , I 9(II):7347-7356.

29. Jacquenet S, Mereau A, Bilodeau PS, Damier L, Stoltzfus CM, Branlant C: A second exon splicing silencer within human immunodeficiency virus type $I$ tat exon 2 represses splicing of Tat mRNA and binds protein hnRNP H. J Biol Chem 200I, 276(44):40464-40475.

30. Cavaloc $Y$, Bourgeois $C F$, Kister L, Stevenin J: The splicing factors 9G8 and SRp20 transactivate splicing through different and specific enhancers. Rna 1999, 5(3):468-483.

31. Darlix JL, Cristofari G, Rau M, Pechoux C, Berthoux L, Roques B: Nucleocapsid protein of human immunodeficiency virus as a model protein with chaperoning functions and as a target for antiviral drugs. Adv Pharmacol 2000, 48:345-372.
32. Cimarelli A, Darlix JL: Assembling the human immunodeficiency virus type I. Cell Mol Life Sci 2002, 59(7): I 166-I I84.

33. Freed EO: Mechanisms of enveloped virus release. Virus Res 2004, 106(2):85-86.

34. Darlix JL, Lopez Lastra M, Mély Y, Roques BP: The nucleocapsid protein at the heart of HIV structure, assembly and CDNA synthesis. HIV Compendium edition. NIAID, AIDS Division. Los Alamos, USA; 2003:69-88.

35. Butsch M, Boris-Lawrie K: Destiny of unspliced retroviral RNA: ribosome and/or virion? J Virol 2002, 76(7):3089-3094.

36. Mangeot PE, Negre D, Dubois B, Winter AJ, Leissner P, Mehtali M, Kaiserlian D, Cosset FL, Darlix JL: Development of minimal lentivirus vectors derived from simian immunodeficiency virus (SIVmac25I) and their use for gene transfer into human dendritic cells. J Virol 2000, 74( I 8):8307-83|5.

37. Bachrach E, Marin M, Pelegrin M, Karavanas G, Piechaczyk M: Efficient cell infection by Moloney murine leukemia virusderived particles requires minimal amounts of envelope glycoprotein. J Virol 2000, 74(18):8480-8486.

38. Maldarelli F, Xiang C, Chamoun G, Zeichner SL: The expression of the essential nuclear splicing factor SC35 is altered by human immunodeficiency virus infection. Virus Res 1998, 53(I):39-5I.

39. Ryo A, Suzuki Y, Arai M, Kondoh N, Wakatsuki T, Hada A, Shuda M, Tanaka K, Sato C, Yamamoto M, Yamamoto N: Identification and characterization of differentially expressed mRNAs in HIV type I-infected human T cells. AIDS Res Hum Retroviruses 2000, 16( 1 0):995-1005.

40. McPhillips MG, Veerapraditsin T, Cumming SA, Karali D, Milligan SG, Boner W, Morgan IM, Graham SV: SF2/ASF binds the human papillomavirus type 16 late RNA control element and is reg. ulated during differentiation of virus-infected epithelial cells. J Virol 2004, 78(19): 10598-10605.

4I. Molin M, Akusjarvi G: Overexpression of essential splicing factor ASF/SF2 blocks the temporal shift in adenovirus premRNA splicing and reduces virus progeny formation. I Virol 2000, 74(1 9):9002-9009.

42. Pelchen-Matthews A, Kramer B, Marsh M: Infectious HIV-I assembles in late endosomes in primary macrophages. / Cell Biol 2003, I62(3):443-455.

Publish with Bio Med Central and every scientist can read your work free of charge

"BioMed Central will be the most significant development for disseminating the results of biomedical research in our lifetime. "

Sir Paul Nurse, Cancer Research UK

Your research papers will be:

- available free of charge to the entire biomedical community

- peer reviewed and published immediately upon acceptance

- cited in PubMed and archived on PubMed Central

- yours - you keep the copyright 